\title{
Psychological morbidity and job satisfaction in hospital consultants and junior house officers: multicentre, cross sectional survey
}

Navneet Kapur, Carol Borrill, Chris Stride

Junior house officers have traditionally been the most distressed doctors in the health service. ${ }^{1}$ Recently, however, there have been reports of significant psychological morbidity in senior doctors such as hospital consultants. ${ }^{2}$ Previous studies found that levels of distress decrease with increasing medical seniority. ${ }^{3}$ Much of the organisational burden of recent hospital reform has fallen on consultants, while junior doctors continue to have their hours of work reduced. We investigated whether these changes had affected the relation between medical seniority, psychological morbidity, and job satisfaction.

\section{Subjects, methods, and results}

We carried out two postal surveys. Questionnaires were sent to all hospital consultants in five UK teaching hospitals as part of the NHS workforce survey. ${ }^{4}$ Questionnaires were also sent to all preregistration medical house officers working in two teaching hospitals in 1995 and 1996. Follow up questionnaires were sent to non-responders, and the house officers also received telephone reminders. We used the 12 item version of the general health questionnaire as a measure of psychological distress. ${ }^{5}$ This can be scored 0 -12, a score of 4 or more identifying a probable case of minor psychiatric disorder such as anxiety or depression. ${ }^{2}$ Each item can also be rated on a three point Likert scale giv- ing a potential score of 0-36, with higher scores representing greater distress. The questionnaire also included validated Likert measures of job satisfaction, work demands, and job autonomy. ${ }^{4}$

The response rate was $60 \%(267 / 445)$ for consultants and $96 \%$ (89/93) for house officers. There was no difference in response rates by specialty or hospital. The consultants were older than the house officers (mean age (SD) 45 (7.8) versus 25.6 (3) years) and a greater proportion were men $(77 \%(206 / 267)$ versus $57 \%(51 / 89)$ women). The table shows the consultants and house officers' scores for various measures; $25 \%$ $(66 / 267)$ of consultants and 19\% (17/89) of house officers scored as cases of psychiatric disorder. For consultants, general health questionnaire scores were most strongly correlated with scores on the work demands scale (Pearsons $+0.42, \mathrm{P}<0.001)$. For house officers, the scores were most strongly correlated with scores on the job autonomy scale (Pearsons $-0.48, \mathrm{P}<0.001$ ).

\section{Comment}

In our study consultants had higher levels of psychological distress and suffered greater demands at work than the house officers. The house officers had lower overall job satisfaction and less job autonomy but were more satisfied with their hours of work.

Non-response among the consultants was a potential source of bias. However, previous studies with

Comparison of job satisfaction, work demands, job autonomy, and general health questionnaire scores for consultants and house officers. Scores on 16 items of job satisfaction are also listed. In all cases higher scores indicate more favourable outcomes except for 12 item version of general health questionnaire (GHQ) and work demands scale where higher scores indicate greater psychological morbidity and increased work demands respectively. Values are mean (SD) unless stated otherwise

\begin{tabular}{|c|c|c|c|c|}
\hline Variable (potential range) & $\begin{array}{c}\text { Consultants* } \\
(\mathrm{n}=267)\end{array}$ & $\begin{array}{l}\text { House officers } \\
(\mathrm{n}=89)\end{array}$ & $\begin{array}{l}\text { Difference in means } \\
(95 \% \mathrm{Cl})\end{array}$ & $\begin{array}{l}\text { P value for } \\
\text { difference }\end{array}$ \\
\hline \multicolumn{5}{|l|}{ Job satisfaction: } \\
\hline Job variety $(1-7)$ & $5.8(1.0)$ & $4.0(1.4)$ & $1.8(1.5$ to 2.1$)$ & $<0.001$ \\
\hline Opportunity to use abilities (1-7) & $5.3(1.3)$ & $4.0(1.3)$ & $1.3(1.0$ to 1.6$)$ & $<0.001$ \\
\hline Amount of responsibility (1-7) & $5.8(1.1)$ & $4.6(1.2)$ & $1.2(0.9$ to 1.5$)$ & $<0.001$ \\
\hline Pay $(1-7)$ & $4.8(1.5)$ & $3.7(1.5)$ & $1.1(0.7$ to 1.5$)$ & $<0.001$ \\
\hline Trust management (1-7) & $4.3(1.2)$ & $3.2(1.4)$ & $1.1(0.8$ to 1.4$)$ & $<0.001$ \\
\hline Management-worker relationships (1-7) & $4.1(1.3)$ & $3.3(1.3)$ & $0.8(0.5$ to 1.1$)$ & $<0.001$ \\
\hline Freedom to choose work method (1-7) & $5.3(1.3)$ & $4.6(1.0)$ & $0.7(0.4$ to 1.0$)$ & $<0.001$ \\
\hline In job training $(1-7)$ & $4.2(1.4)$ & $3.6(1.5)$ & $0.6(0.2$ to 1.0$)$ & 0.002 \\
\hline Attention paid to suggestions (1-7) & $4.3(1.3)$ & $4.0(1.2)$ & $0.3(-0.04$ to 0.6$)$ & 0.09 \\
\hline Recognition for good work (1-7) & $4.3(1.5)$ & $4.1(1.3)$ & $0.2(-0.1$ to 0.5$)$ & 0.35 \\
\hline Fellow workers (1-7) & $5.5(1.1)$ & $5.5(1.0)$ & $0(-0.2$ to 0.2$)$ & 0.93 \\
\hline Job security $(1-7)$ & $5.5(1.1)$ & $5.6(1.0)$ & $-0.1(-0.3$ to 0.1$)$ & 0.32 \\
\hline Working conditions (1-7) & $4.2(1.5)$ & $4.4(1.3)$ & $-0.2(-0.55$ to 0.15$)$ & 0.22 \\
\hline Chance of promotion (1-7) & $4.4(1.4)$ & $4.7(1.0)$ & $-0.3(-0.65$ to 0.05$)$ & 0.053 \\
\hline Your immediate boss (1-7) & $4.9(1.4)$ & $5.4(1.2)$ & $-0.5(-0.8$ to -0.2$)$ & 0.001 \\
\hline Hours of work (1-7) & $3.7(1.6)$ & $4.2(1.6)$ & $-0.5(-0.9$ to -0.1$)$ & 0.006 \\
\hline Work demands (6-30) & $21.4(6.0)$ & $14.9(4.4)$ & 6.5 (5.2 to 7.8$)$ & $<0.001$ \\
\hline Total job satisfaction (16-112) & $74.4(11.8)$ & $69.1(10.2)$ & 5.3 (2.4 to 8.3$)$ & $<0.001$ \\
\hline Job autonomy (6-30) & $23.9(4.5)$ & $19.0(3.5)$ & $4.9(3.8$ to 6.0$)$ & $<0.001$ \\
\hline GHQ 12 score $(0-36)$ & $12.1(4.7)$ & $9.5(4.5)$ & $2.6(1.5$ to 3.7$)$ & 0.002 \\
\hline
\end{tabular}

${ }^{*}$ Consultants from different specialties considered together as no difference in GHQ scores or job satisfaction. $\dagger t$ test for independent samples.

Department of Psychiatry and Behavioural Sciences, Rawnsley Building,

Manchester Royal

Infirmary,

Manchester M13 9WL

Navneet Kapur, lecturer in psychiatry

Institute of Work

Psychology,

University of Sheffield, Sheffield S10 2TN

Carol Borrill, project manager Chris Stride, statistician

Correspondence to: Dr Kapur

BMJ 1998;317:511-2 
higher response rates have reported similar levels of distress. ${ }^{2}$ A study carried out as part of the NHS workforce survey found no difference in the levels of distress between responders and non-responders, ${ }^{4}$ suggesting that non-response was not systematically related to psychological health. Our results may not be generalisable to non-teaching hospitals and might be confounded by the fact that consultants and house officers were selected from different trusts. In fact, one hospital was a source of both consultants and house officers for this study, and the occupational group differences at this hospital remained significant.

Some of these data are three years old. The continued upheaval in the health service and further reductions in junior house doctors' working hours mean that the differences in psychological distress between senior hospital doctors and their most junior colleagues may now be more pronounced. One approach to addressing this problem could be to tackle specific sources of occupational stress in different groups. For example, our results suggest consultants might benefit from a reduction in their workload, while house officers might benefit if they had greater autonomy.
We would like to thank the consultants and house officers.

Contributors: NK coordinated and collected data for the house officer study, helped analyse the data, and wrote the initial draft of the paper. $\mathrm{CB}$ coordinated the consultant study and commented on drafts of the paper. CS helped to extract and analyse the data, and commented on drafts of the paper. NK and $\mathrm{CB}$ will act as guarantors for the paper.

Funding: The NHS workforce survey was funded under the mental health programme of the NHS executive (Northern and Yorkshire).

Conflict of interest: None.

1 Firth-Cozens J. Emotional distress in junior house officers. BMJ 1987;295:533-6.

2 Ramirez AJ, Graham J, Richards MA, Cull A, Gregory WM. Mental health of hospital consultants: the effects of stress and satisfaction at work. Lancet 1996;347:724-8

3 Hsu K, Marshall V. Prevalence of depression and distress in a large sample of Canadian residents, interns, and fellows. Am J Psych 1987;144:1561-6.

4 Borrill CF, Wall TD, West MA, Hardy GE, Shapiro DA, Carter A, et al. Mental health of the workforce in NHS trusts: phase I final report. Sheffield: Institute of Work Psychology, University of Sheffield, and Leeds: Department of Psychology, University of Leeds, 1996.

5 Goldberg DP. The detection of psychiatric illness by questionnaire. London: Oxford University Press, 1972

(Accepted 5 May 1998)

\title{
The quality and stability of essential drugs in rural Zimbabwe: controlled longitudinal study
}

\author{
Hanif Nazerali, Hans V Hogerzeil
}

Zimbabwe Essential Drugs Action Programme, PO Box CY 924, Harare, Zimbabwe Hanif Nazerali, pharmacist

World Health Organisation, Action Programme on Essential Drugs, 1211 Geneva, Switzerland

Hans V Hogerzeil, medical officer

Correspondence to: Dr Hogerzeil hogerzeilh@who.ch

BMJ 1998;317:512-3
In 1988 an alarming WHO report of substandard ergometrine injection in three developing countries ${ }^{1}$ led to field studies on the stability of essential drugs during international transport to the tropics and to specific stability studies on oxytocic drugs. ${ }^{2}$ We performed a controlled longitudinal study to measure the quality of essential drugs within rural Zimbabwe and to determine whether any failure was due to poor initial quality or to instability of the drugs during inland distribution and storage.

\section{Subjects, methods, and results}

During a two year period (January 1991 to December 1992), samples of drugs arriving at central medical stores in Harare and Bulawayo were retained as controls, and samples of the same batches were later retrieved from hospitals and health centres in five remote, hot, rural districts. We studied 789 samples of 26 brands of 13 essential drugs (selected for relevance to public health and suspected stability problems); 176 samples were taken from central stores and 613 from rural facilities. Of the latter, 261 samples were matched with central samples of the same batch to make longitudinal pairs. Drug quality was measured by level of active ingredient as percentage of stated content and by compliance (pass/fail) with assay standards of the British Pharmacopoeia. Drug stability was measured by comparing mean assay values at central and rural level and by paired analysis of central and rural samples of the same batch.

Drug quality in rural facilities was satisfactory for injectable benzylpenicillin and oral forms of acetylsalicylic acid, amoxicillin, ampicillin, doxycycline, ferrous sulfate, phenylmethoxypenicillin, and tetracycline; it was indeterminate for epinephrine injection. Poor initial quality accounted for problems in injectable ampicillin (2/10 central samples failed, with $87 \%$ and $91 \%$ content) and retinol tablets (5/5 failed; mean assay $73 \%, 95 \%$ confidence interval $63 \%$ to $83 \%$ ), with similar results at rural facilities. An aqueous formulation of injectable procaine benzylpenicillin showed moderate instability with $4 \%(1 \%$ to $6 \%)$ loss after 4.3 months but the assay remained within pharmacopoeial limits. Poor initial quality of all three brands of ergometrine injec-

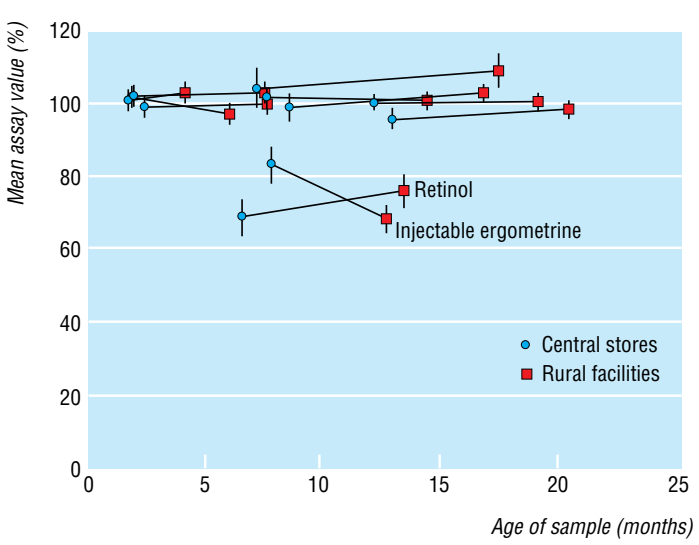

Stability of essential drugs in Zimbabwe. Matched pairs from same batch of retinol (gain) and ergometrine (loss) were unstable acetylsalicylic acid, amoxicillin, ampicillin, benzylpenicillin, doxycycline, epinephrine, ferrous sulfate, phenoxymethylpenicillin, procaine benzylpenicillin, and tetracycline were stable 